\title{
Identification of the Nodose Ganglia and TRPV1 in Swine
}

\author{
Don Hayes Jr. • Kathleen K. Nicol • Joseph D. Tobias • Louis G. Chicoine • \\ Victoria L. Duffy $\cdot$ Heidi M. Mansour · Thomas J. Preston
}

Received: 14 June 2013/Accepted: 8 July 2013/Published online: 28 July 2013

(C) Springer Science+Business Media New York 2013

The vagal nerve is the tenth cranial nerve $(\mathrm{CN})$ and is the only $\mathrm{CN}$ to extend through the neck into the thorax and abdomen. As a component of the autonomic nervous system, the vagal nerve innervates the visceral organs, including the lung. The majority of the bronchopulmonary sensory nerves are unmyelinated (C-fiber) afferents of the vagal nerve. The cell bodies of the visceral afferent fibers of the vagus nerve are located bilaterally in the inferior ganglion-also termed the nodose ganglia (NG). More recently, transient receptor potential vanilloid type 1 (TRPV1) channels have been identified and are expressed on the vagal C-fiber afferents, which play an important role in the cholinergic reflex pathway [1-3]. TRPV1 channels are nonselective cation channels and polymodal transducers that

The study was approved by the Institutional Animal Care and Use Committee (IACUC) of the Nationwide Children's Hospital with strict adherence to the IACUC guidelines regarding humane use of animals.

The work and subsequent manuscript was completed at the Nationwide Children's Hospital and The Ohio State University, Columbus, $\mathrm{OH}$.

D. Hayes Jr. $(\bowtie) \cdot$ L. G. Chicoine

Department of Pediatrics, Nationwide Children's Hospital, The Ohio State University College of Medicine, 700 Children's Drive, Columbus, OH 43205, USA

e-mail: hayes.705@osu.edu

K. K. Nicol

Department of Pathology, Nationwide Children's Hospital, The Ohio State University College of Medicine,

Columbus, $\mathrm{OH}$, USA

J. D. Tobias

Department of Anesthesiology and Pain Medicine, Nationwide Children's Hospital, The Ohio State University College

of Medicine, Columbus, OH, USA are known to be activated by increasing temperature, acidity (hydrogen ion), and endogenous inflammatory mediators [13].

Although differences exist between swine and human anatomy, the patterns of the vagal nerve are largely similar and closer in size than standard rodent models [4]. Data are limited on identifying autonomic neurons and associated cell bodies, including the NG in swine $[5,6]$. The importance and study of pulmonary neurobiology in acute lung injury is gaining momentum and often requires the use of large animal models, including swine. With no previous reports of the identification and subsequent histopathologic confirmation of the NG and TRPV1 in swine, we provide our experiences in identifying the NG in common crossbred piglets used in an acute lung injury model investigating the benefits of extracorporeal membrane oxygenation. The vagal nerve was dissected based on anticipated location. Figure 1 outlines the two anatomical patterns of the vagal laryngeal branches as previously described by Ding et al. [4]. Figure 2 illustrates the NG structure of one animal on gross examination with this animal having anatomical pattern A from Fig. 1. Histopathologic assessment confirmed the NG on hematoxylin

\section{L. Duffy · T. J. Preston}

Department of Cardiovascular Perfusion, The Heart Center, Nationwide Children's Hospital, Columbus, OH, USA

\section{H. M. Mansour}

Skaggs Center of Pharmaceutical Sciences, College of Pharmacy, The University of Arizona-Tucson, Tucson, AZ, USA 
Fig. 1 Two anatomical patterns of the vagal laryngeal branches in swine. a $C L N$ cranial laryngeal nerve arising from the $N G$ nodose ganglion with subsequent bifurcation into the $I L N$ internal laryngeal nerve and $E L N$ external laryngeal nerve. b Both the ILN and ELN branches directly from the cervical vagal nerve without a CLN trunk
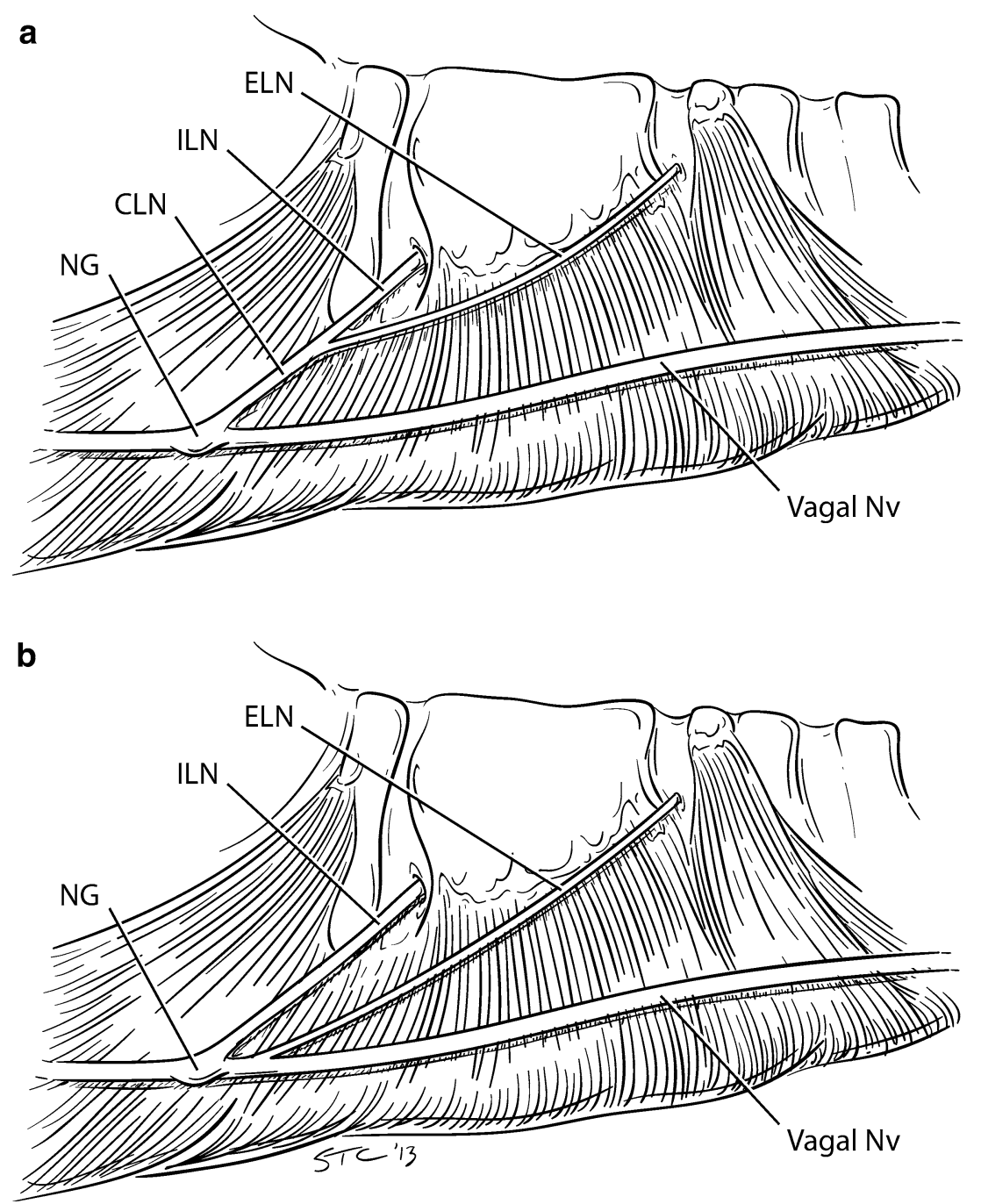

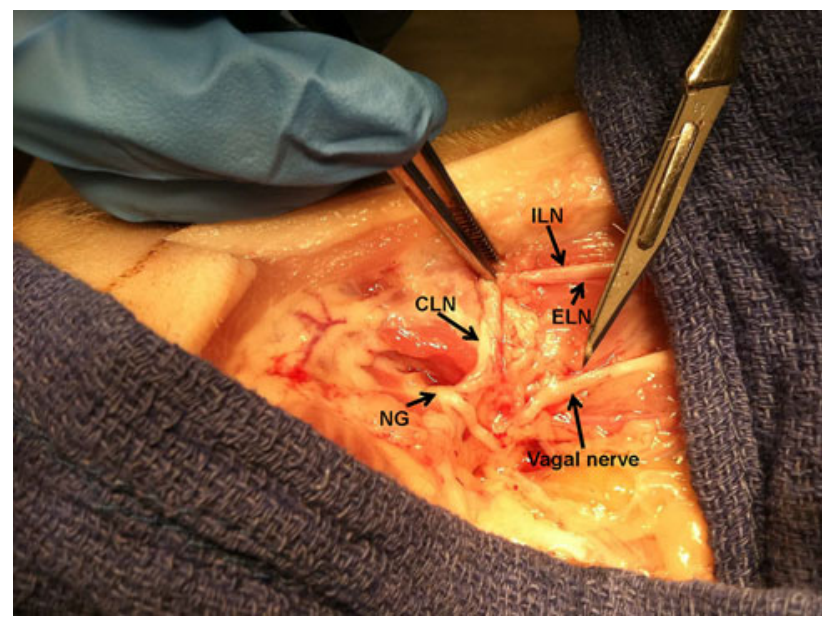

Fig. 2 Gross examination of this animal demonstrating the vagal laryngeal nerve branches in an anatomic pattern that would be type A as illustrated in Fig. 1. The $C L N$ cranial laryngeal nerve arises from the $N G$ nodose ganglion that bifurcates into the $I L N$ internal laryngeal nerve and ELN external laryngeal nerve. Incisions were performed in the dorsal neck, 4-6 $\mathrm{cm}$ inferior to the skull base

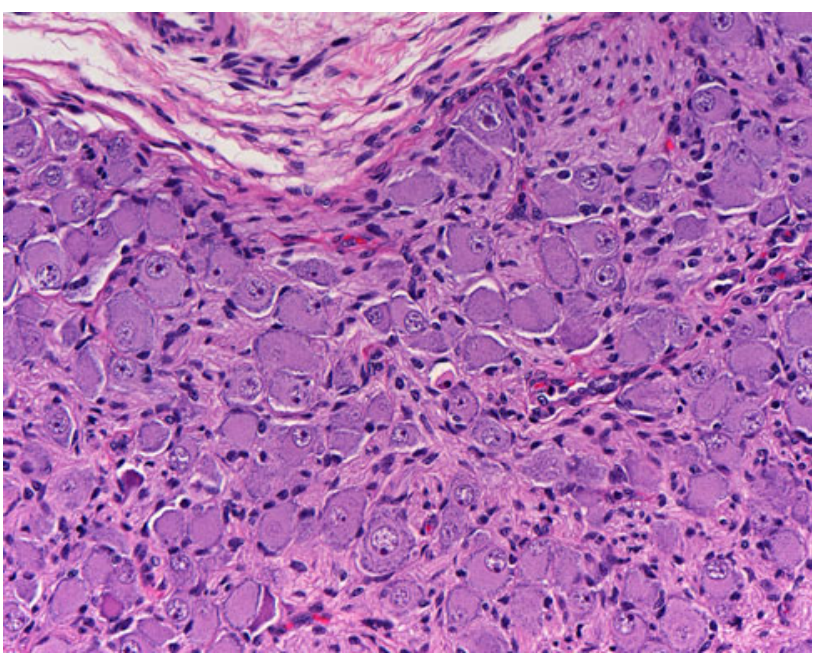

Fig. 3 A representative section of the vagal nerve nodose ganglion stained with $H \& E$ hematoxylin and eosin with associated nerve fibers in the adjacent soft tissue (magnification $\times 20$ ) 


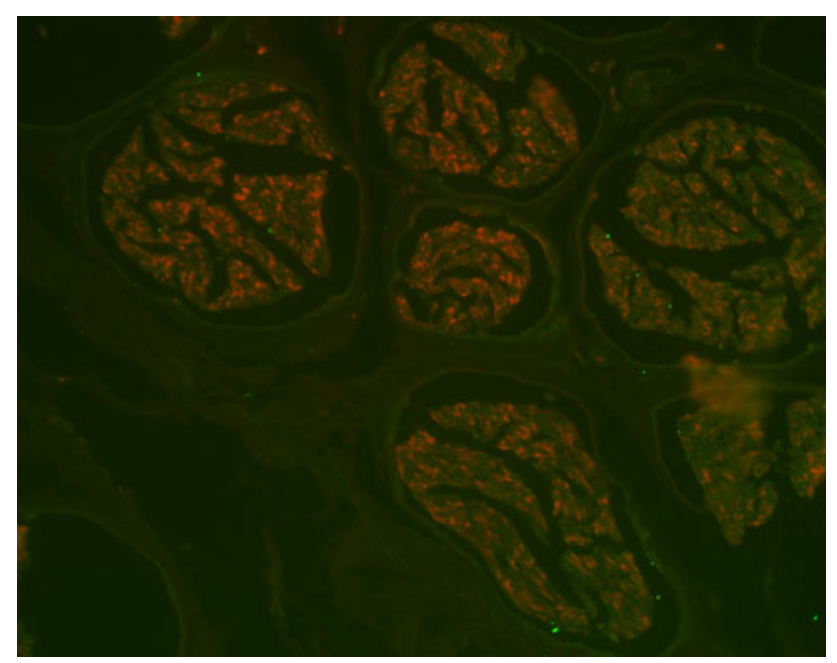

Fig. 4 Immunofluorescence staining of TRPV1 channels in the nodose ganglion of swine (magnification $\times 20$ )

and eosin (H\&E) staining (Fig. 3) and immunofluorescence staining for TRPV1 channels (Fig. 4).

Nodose ganglia were successfully found with TRPV1 being identified in an efficient manner despite no previously reported methodology. Researchers in this area need to be aware of the anatomical variances of the vagal nerve and its laryngeal branches in swine. Future work is planned to address the neurobiology of the respiratory system during acute lung injury in this large animal model.
Acknowledgments The authors thank Stacy Turpin for her assistance in the development of the illustration used in this manuscript.

Funding Funding that supported this work was provided by The Research Institute of Nationwide Children's Hospital.

Conflict of interest The authors have no conflict of interest or disclosures related to any companies or organizations whose products or services that are discussed in this manuscript.

\section{References}

1. Yamamoto Y, Sato Y, Taniguchi K (2007) Distribution of TRPV1and TRPV2-immunoreactive afferent nerve endings in rat trachea. J Anat 211(6):775-783

2. Gu Q, Lin RL, Hu HZ, Zhu MX, Lee LY (2005) 2-Aminoethoxydiphenyl borate stimulates pulmonary $\mathrm{C}$ neurons via the activation of TRPV channels. Am J Physiol Lung Cell Mol Physiol 288(5):L932-L941

3. Lee LY, Ni D, Hayes D Jr, Lin RL (2011) TRPV1 as a cough sensor and its temperature-sensitive properties. Pulm Pharmacol Ther 24(3):280-285

4. Ding P, Tufano RP, German RZ (2012) Anatomical anomalies of the laryngeal branches of the vagus nerve in pigs (Sus scrofa). Lab Anim 46(4):338-340

5. Hopkins DA, Gootman PM, Gootman N, Armour JA (1997) Anatomy of medullary and peripheral autonomic neurons innervating the neonatal porcine heart. J Auton Nerv Syst 64(2-3):74-84

6. Philippe C, Cuber JC, Bosshard A, Rampin O, Laplace JP, Chayvialle JA (1990) Galanin in porcine vagal sensory nerves: immunohistochemical and immunochemical study. Peptides 11(5): 989-993 